\title{
An Analytical Method to Predict the Static Performance of a Planar Actuator
}

\author{
Aly F. Flores Filho, Altamiro A. Susin, and Marilia A. da Silveira
}

\begin{abstract}
An analytical method for predicting the three-dimensional (3-D) magnetic flux density distribution in the air gap produced by permanent magnets of an electromagnetic planar actuator, was developed. The analytical 3-D model was validated by means of a finite-element model and by measurements. It allowed the understanding of the behavior of the flux density in the air gap and predicting the values of the involved forces.
\end{abstract}

Index Terms-Analytical model of the air gap magnetic field, planar actuator, static forces.

\section{INTRODUCTION}

A N ANALYTICAL method for predicting the three-dimensional (3-D) flux density distribution in the air gap of an electromagnetic planar actuator and its propulsion planar force, was developed. Fig. 1 shows a perspective of the prototype under study. The stator has multiphase armature windings placed in such a way that two orthogonal windings are produced: one is assembled to form the $x$ coil phases and the other, the $y$ coil phases. The armature core is a slotless iron slab. The wireless mover has two NdFeB-type permanent magnets. The mover is attached to a suspension structure with linear bearings that enable bidirectional motion. This results in an actuator with two degrees of freedom. The movement can take place along the $x$ axis and $y$ axis simultaneously. When one permanent magnet is located over an excited coil phase, a magnetic propulsion force will be created on the mover and that pushes the latter accordingly. The intensity and direction of the resulting magnetic force depend on the values of the active coil magnetomotive force and the air gap flux density established by the permanent magnets.

\section{Analytical Model of the Magnetic Flux Density in THE AIR GAP PRODUCED BY THE PERMANENT MAGNETS}

In order to obtain the field equation in the air gap due to the permanent magnets, the authors developed an analytical model. The method is based on an analysis of a 3-D model in rectangular coordinates. The magnetic field due to the permanent magnets is analyzed separately, not taking into account the field produced by the armature winding. So, the regions under study are considered free from electric currents and the magnetic field, $\vec{H}$, can be obtained by using the gradient of the magnetic scalar potential, $\psi$, according to $\vec{H}=-\nabla \psi$. The equation of the mag-

Manuscript received January 6, 2003.

A. F. Flores Filho and A. A. Susin are with the Federal University of Rio Grande do Sul, Porto Alegre, RS, 90.035-190, Brazil (e-mail: flores@eletro.ufrgs.br; susin@ eletro.ufrgs.br).

M. A. da Silveira is with the Lutheran University of Brazil, Canoas, RS, 90.420-280, Brazil (e-mail: marilia@ eletro.ufrgs.br).

Digital Object Identifier 10.1109/TMAG.2003.816258

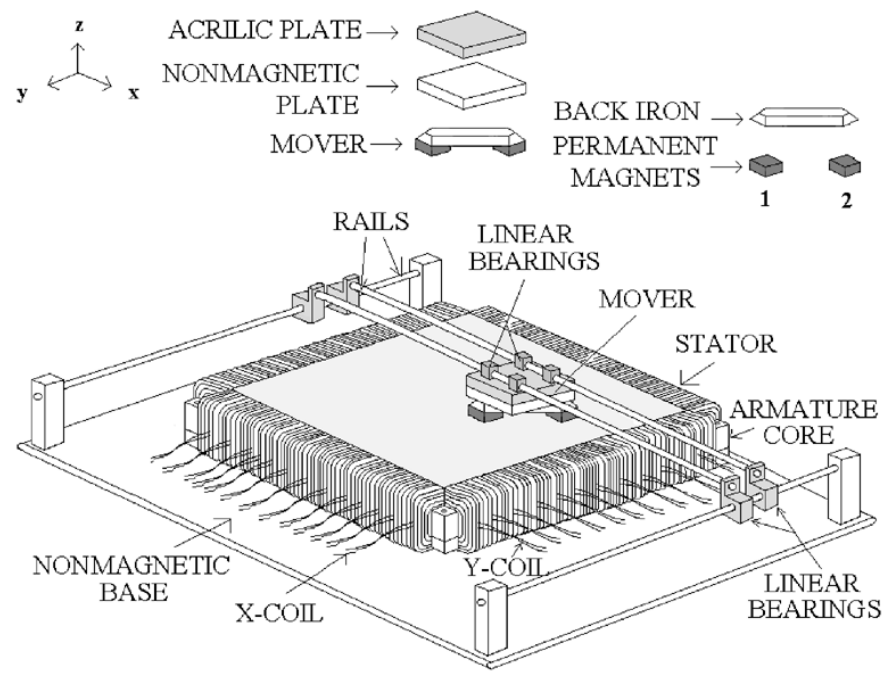

Fig. 1. General 3-D view of the planar actuator under study.

netic scalar potential in the air gap was obtained by means of the Laplace's equation, $\nabla^{2} \psi=0$. A solution method to that equation involves the determination of a field function, which satisfies the Laplace's equation, the imposed boundaries and the field conditions in the permanent magnets region [1], [2]. Fig. 2(a) and (b) show the frontal and the upper views of the geometry under study, where $l_{g}$ is the air gap length, $l_{m}$ is the permanent magnets length and $l_{d}$ corresponds to the half of the side of the square polar area of each permanent magnet. The boundary conditions were set in such way that the magnetic scalar potential is equal to zero on the planes $x=0, y=0$, $x=l_{t} / 2, y=l_{t} / 2, x=l_{t}$ and $y=l_{t}$, where $l_{t}$ corresponds to the side of the square area considered to the analysis. In order to obtain the magnetic scalar potential, the magnetic circuit of the planar actuator was divided into regions. Fig. 2(c) presents the regions and the boundaries, denoted by $O, B, G$, and $P$. Only the effects of one permanent magnet are taken into account, as long as the symmetry is present. As the magnetic field due to the current in the windings is not considered by the present analysis, the region between the boundaries $O$ and $B$ has the same magnetic properties as the air.

Applying the boundary conditions to the Laplace's equation, the magnetic scalar potential in the air gap, $\psi_{g}$, can be represented by means of a double Fourier series, according to the expression:

$$
\psi_{g}(x, y, z)=\sum_{\substack{n=1,3, \ldots \\ m=1,3, \ldots}}^{\infty}\left(k_{1} e^{\gamma z}+k_{2} e^{-\gamma z}\right) \sin (\kappa n x) \sin (\kappa m y)
$$




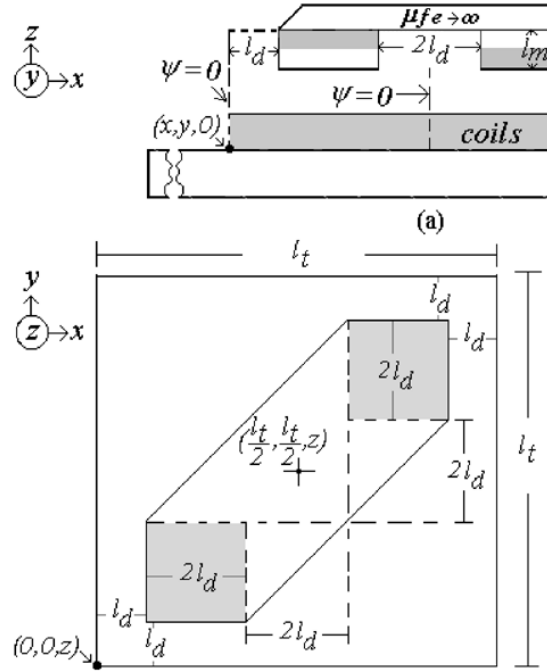

(b)
不

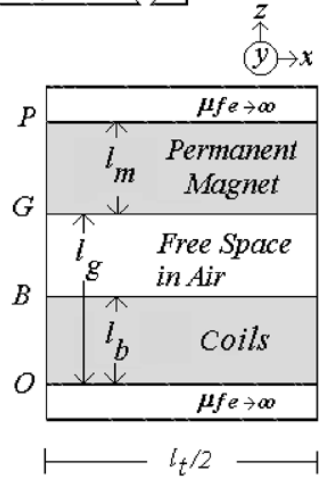

(c)
Fig. 2. (a) Frontal and (b) upper views of the model under study and (c) its regions and boundaries.

In (1), $n$ and $m$ are integers, $\kappa$ is defined by $\kappa=2 \pi / l_{t}$ and $\gamma$ by $\gamma=\left(2 \pi / l_{t}\right) \sqrt{n^{2}+m^{2}}$. Still in (1), $k_{1} e^{\gamma z}+k_{2} e^{-\gamma z}$ corresponds to $\psi_{g_{n, m}}$, the set of coefficients of Fourier series. In the mover, the two permanent magnets are placed in an anti-parallel way with respect to each other. For each one, the magnetization vector, $\vec{M}$, has only one component through the $z$ axis. So, $\vec{M}$ can be represented by $M_{z} \vec{k}$ and $M_{z}$ is given by

$$
\begin{array}{r}
M_{z}=\sum_{\substack{n=1,3, \ldots \\
m=1,3, \ldots}}^{\infty}\left(\frac{16 M_{o}}{\pi^{2}} \frac{\cos \left(\kappa n l_{d}\right) \cos \left(\kappa m l_{d}\right)}{n m}\right) \\
\cdot \sin (\kappa n x) \sin (\kappa m y) .
\end{array}
$$

In (2), $M_{O}$ is the remanent magnetization of the permanent magnets. In the permanent magnet region, the magnetic scalar potential must satisfy the Poisson's equation, $\nabla^{2} \psi=\nabla \vec{M}$. So, the magnetic scalar potential is represented by $\psi=\psi_{h}+\psi_{p}$, where $\psi_{h}$ is a homogeneous term and must satisfy the Laplace's equation and $\psi_{p}$ is the particular term, solved by Poisson's equation [3], [4]. Equation (2) allows one to conclude that the divergence of $\vec{M}$ is equal to zero. So, the expression of the magnetic scalar potential in the permanent magnet region is equal to

$\psi_{p m}(x, y, z)=\sum_{\substack{n=1,3, \ldots \\ m=1,3, \ldots}}^{\infty}\left(k_{3} e^{\gamma z}+k_{4} e^{-\gamma z}\right) \sin (\kappa n x) \sin (\kappa m y)$.

It remains to find the values of the constants $k_{1}, k_{2}, k_{3}$ and $k_{4}$ in (1) and (3). Owing to the assumption of infinity permeability of stator iron, the magnetic scalar potential on the boundary $O$, where $z=0$, is equal to zero. The expression of the magnetic scalar potential in the air gap on the boundary $O, \psi_{g}^{O}$ (the superscript denotes the boundary), is given by

$$
\psi_{g}^{O}=\sum_{\substack{n=1,3, \ldots \\ m=1,3, \ldots}}^{\infty}\left(k_{1} e^{0}+k_{2} e^{0}\right) \sin (\kappa n x) \sin (\kappa m y) .
$$

Again, owing to the assumption of infinity permeability of the back iron, the magnetic scalar potential on the boundary $P$, where $z=\left(l_{g}+l_{m}\right)$, is equal to zero; and the expression of the magnetic scalar potential in the permanent magnet region on the boundary $P, \psi_{p m}^{P}$, is

$$
\begin{aligned}
& \psi_{p m}^{P}= \sum_{\substack{n=1,3, \ldots \\
m=1,3, \ldots}}^{\infty}\left(k_{3} e^{\gamma\left(l_{g}+l_{m}\right)}+k_{4} e^{-\gamma\left(l_{g}+l_{m}\right)}\right) \\
& \cdot \sin (\kappa n x) \sin (\kappa m y) .
\end{aligned}
$$

On the boundary $G$, where $z=l_{g}$, the $z$ component of the magnetic fields in the air gap and in the permanent magnets are related by $H_{g_{z}}^{G}=H_{p m_{z}}^{G}+M_{z}$ and the respective magnetic scalar potentials are related by $\psi_{g}^{G}=\psi_{p m}^{G}$. The $z$ component of the magnetic field $\vec{H}$ is obtained from $H_{z}=-\partial \psi / \partial z$. The expressions for $H_{g_{z}}^{G}$ and $H_{p m_{z}}^{G}$ are given, respectively, by

$$
H_{g_{z}}^{G}=\sum_{\substack{n=1,3, \ldots \\ m=1,3, \ldots}}^{\infty} \gamma\left(-k_{1} e^{\gamma l_{g}}+k_{2} e^{-\gamma l_{g}}\right) \sin (\kappa n x) \sin (\kappa m y)
$$

and

$$
H_{p m_{z}}^{G}=\sum_{\substack{n=1,3, \ldots \\ m=1,3, \ldots}}^{\infty} \gamma\left(-k_{3} e^{\gamma l_{g}}+k_{4} e^{-\gamma l_{g}}\right) \sin (\kappa n x) \sin (\kappa m y)
$$

Applying those conditions to (1) and (3), after some operations, one can obtain a set of four equations and four unknowns, which allows one to solve for the desired potentials. The $z$ component of the magnetic flux density, $B_{g_{z}}$, is given by

$$
\begin{array}{r}
B_{g_{z}}=\mu_{O} \frac{16 M_{o}}{\pi^{2}} \sum_{\substack{n=1,3, \ldots \\
m=1,3, \ldots}}^{\infty} \cosh (\gamma z) \frac{\left(e^{\gamma l_{g}}-e^{\gamma\left(2 l_{m}+l_{g}\right)}\right)}{\left(e^{2 \gamma\left(l_{m}+l_{g}\right)}-1\right)} \\
. \frac{\cos \left(\kappa n l_{d}\right) \cos \left(\kappa m l_{d}\right)}{n m} \sin (\kappa n x) \sin (\kappa m y) .
\end{array}
$$

\section{The Equation of the Planar Propulsion Force}

The force that produces movement over the plane depends on the current density in the armature windings and the $z$ component of the magnetic flux density produced by the permanent magnets, $B_{g_{z}}$. The force that acts over one of the permanent magnet is obtained from

$$
\begin{aligned}
\vec{F}_{N} & =\int_{V_{N}}-\left(\vec{J}_{N} \times \vec{B}_{g_{N}}\right) d V_{N} \\
& =\int_{V_{N}} B_{g_{z_{N}}}\left(-J_{y_{N}} \bar{i}+J_{x_{N}} \bar{j}\right) d V_{N} \\
& =-F_{x_{N}} \bar{i}+F_{y_{N}} \bar{j} .
\end{aligned}
$$

where $F_{N}$ is the propulsion force produced over the permanent magnet $N, J_{y_{N}}$, and $J_{x_{N}}$ are the current density in the $x$ and $y$ coil phases, respectively, and $V_{N}$ is the volume of integration that surrounds the active coil phases. Applying (8) to (9), one 


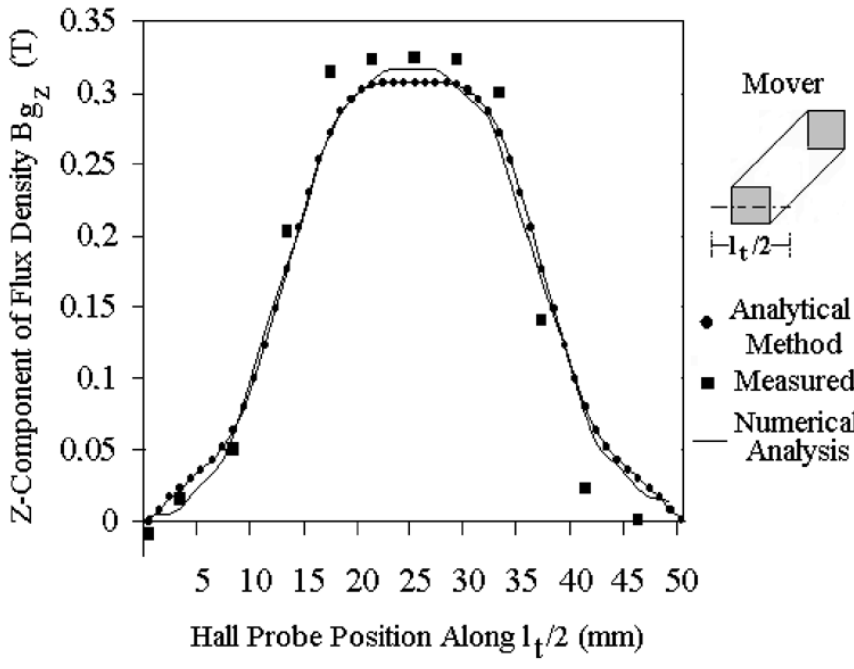

(a)

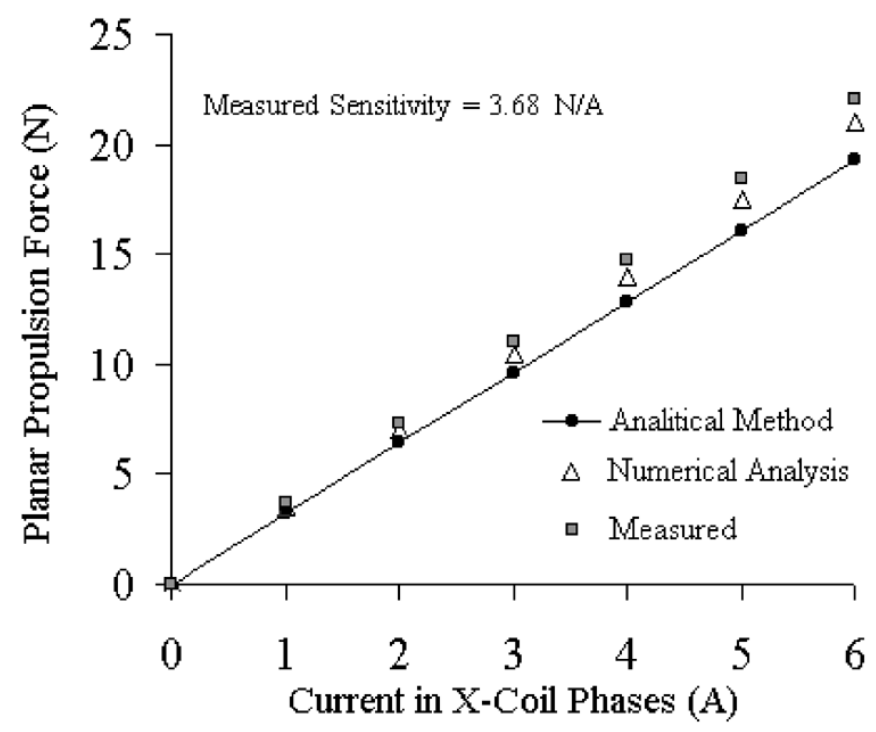

(b)

Fig. 3. (a) Graph of the $z$ component of flux density in the air gap vs. position on the plane, when current in the phases of the armature is zero and (b) graph of the planar propulsion force vs. current in the $x$ coil phases located under the permanent magnets.

can obtain the equation of the planar propulsion force that acts over one permanent magnet:

$$
\begin{aligned}
\vec{F}_{N}=\sum_{\substack{n=1,3, \ldots \\
m=1,3, \ldots}}^{\infty} \sinh \gamma l_{g} \frac{\left(e^{\gamma l_{g}}-e^{\gamma\left(2 l_{m}+l_{g}\right)}\right)}{\left(e^{2 \gamma\left(l_{m}+l_{g}\right)}-1\right)} \\
\cdot \frac{8 l_{t}^{3} \mu_{o} M_{o}}{\pi^{5} n^{2} m^{2} \sqrt{n^{2}+m^{2}}} \cos \left(\kappa n l_{d}\right) \cos \left(\kappa m l_{d}\right) \\
\cdot\left(-J_{y_{N}} \cos \left(\kappa n l_{d}\right) \vec{i}+J_{x_{N}} \cos \left(\kappa m l_{d}\right) \vec{j}\right) .
\end{aligned}
$$

\section{RESULTS AND CONCLUSIONS}

Fig. 3(a) presents the graph of the $z$ component of flux density in the air gap versus position on the plane underneath the mover
TABLE I

VALUes of the $z$ COMPONENT OF THE FluX DENSITY IN THE AIR GAP

\begin{tabular}{lccc}
\hline \hline$B_{g_{z}}$ & Analytical Method & Numerical $\Lambda$ nalysis & Measured \\
\hline Peak Value (T) & 0.307 & 0.317 & 0.324 \\
Dißrerence \% & 5.25 & 2.16 & 0 \\
M.iean Value (T) & 0.161 & 0.163 & 0.167 \\
Difference \% & 3.59 & 2.40 & 0 \\
\hline \hline
\end{tabular}

TABLE II

VAlues of the $x$ Component Of THe Planar PRopulsion Force

\begin{tabular}{lccc}
\hline Planar Force & $\mathrm{I}=3.0 \mathrm{~A}$ & $\mathrm{I}=6.0 \mathrm{~A}$ & Average Sensitivity (N/A) \\
\hline Analytical Method & $9.61 \mathrm{~N}$ & $19.26 \mathrm{~N}$ & 3.21 \\
Numerical Analysis & $10.49 \mathrm{~N}$ & $21.01 \mathrm{~N}$ & 3.48 \\
Measured & $11.30 \mathrm{~N}$ & $22.08 \mathrm{~N}$ & 3.68 \\
\hline \hline
\end{tabular}

at $z=8 \mathrm{~mm}$, when current in the phases of the armature is zero. Fig. 3(b) presents the graph of the planar propulsion force that acts over the mover when $x$ coil phases are fed with current. The graphs present values obtained by the analytical method described in this paper, by means of (8) and (10), respectively. For comparison and validation of the analytical model, figures are obtained by numerical analysis and from measurements, under the same conditions of current and position of those obtained by the analytical method. The measured values of flux density were obtained by means of a Gaussmeter and a Hall effect probe. The measured values of planar propulsion force were obtained by means of load cells. The numerical values are the results of a finite element analysis. The values in (8) were computed considering the odd harmonics up to the ninth order. Table I shows the values of the $z$ component of the flux density in the air gap related to the Fig. 3(a). Table II presents the values of the $x$ component of the propulsion planar force related to Fig. 3(b). The theoretical values resulted smaller than the measured ones possibly because the authors used the magnetic characteristics of the permanent magnets obtained from catalogues. The analytical results showed a good agreement to those obtained by numerical analysis and tests and allowed to foreseen the behavior of the propulsion force as a function of the current density in the armature winding. The measured sensitivity of the planar actuator is equal to $3.68 \mathrm{~N} / \mathrm{A}$.

\section{REFERENCES}

[1] J. R. Melcher, Continuum Electromechanics. Cambridge, MA: MIT Press, 1981.

[2] K. J. Binns, P. J. Lawrenson, and C. W. Trowbridge, Electric and Magnetic Fields. West Sussex, U.K.: Wiley, 1992.

[3] W. J. Kim, "High-precision planar magnetic levitation," Ph.D. dissertation, MIT, Cambridge, MA, 1997.

[4] D. L. Trumper, M. E. Williams, and T. H. Nguyen, "Magnet arrays for synchronous machines," in Proc. IEEE IAS 28th Annu. Meeting, 1993, pp. 216-223. 